\title{
CDHA Ceramic Microspheres for Periodontitis Treatment: Synthesis, Characterization and Doxycycline Release Profiles
}

\author{
Sunita Prem Victor \\ TS Sampath Kumar \\ Department of Metallurgical and Materials Engineering \\ Indian Institute of Technology Madras, Chennai 600036, India \\ E-mail:tssk@iitm.ac.in \\ Received March 1, 2011 ; Accepted April 1, 2011
}

\begin{abstract}
The present study is focused on the development of doxycycline loaded calcium-deficient hydroxyapatite (CDHA) microspheres for the treatment of periodontitis. The CDHA microspheres were formed by liquid immiscibility effect using gelatin and paraffin oil with varying $\mathrm{Ca} / \mathrm{P}$ ratios using calcium hydroxide and diammonium hydrogen orthophosphate as precursors. The morphology of the microspheres as characterized by SEM was optimized by varying the gelatin content. The doxycycline incorporation and its release profiles were studied by UV-Visible spectroscopy in phosphate buffer at physiological conditions. The pH of the buffer solution was initially optimized to have maximum amount of drug loading. Doxycycline loading around the physiological pH of 7 has the highest amount of drug incorporation. All the microspheres exhibit similar release profiles with an initial gradual increase reaching a maximum value and then nearly constant release. The microspheres formed using $6 \%$ gelatin shows maximum amount of drug release of $80 \%$.
\end{abstract}

Keywords: Resorbable ceramics, periodontitis, CDHA, doxycycline, microspheres, drug delivery.

\section{Introduction}

The poor blood circulation in osseous tissue is the main cause of the reduced therapeutic effects of administered drugs at the site of bone infections and necessitates the supply of large doses of antibiotics systematically to achieve clinically favorable results ${ }^{1}$. Ceramic particulate delivery systems with incorporated drugs are evaluated as an alternative for effective treatment of bone diseases ${ }^{2}$. Recently novel strontium substituted calcium phosphate cement loaded with doxycycline hyclate has also been used to elucidate the effect of strontium substitution on antibiotic delivery ${ }^{3}$. Delivery of active agents by resorbable bioceramics such as tricalcium phosphate (TCP), calcium-deficient hydroxyapatite (CDHA) and biphasic calcium phosphate (BCP) ceramics (consisting of hydroxyapatite (HA) and TCP phases) have been frequently reported for orthopedic uses ${ }^{4,5}$. The mineral component of bone is essentially CDHA which is structurally similar to stoichiometric $\mathrm{HA}$ $(\mathrm{Ca} / \mathrm{P}=1.67)$ but compositionally has a $\mathrm{Ca} / \mathrm{P}$ ratio of about 1.5 which is similar to TCP. The CDHAs with $\mathrm{Ca} / \mathrm{P}$ ratios ranging from 1.67 to 1.33 originate from the loss of $\mathrm{Ca}^{2+}$ ions from the unit cell and hence the name calcium deficient hydroxyapatite with the formula Ca ${ }_{10-x}\left(\mathrm{HPO}_{4}\right)_{x}\left(\mathrm{PO}_{4}\right)_{6-x}(\mathrm{OH})_{2-x}{ }^{6}$. 
Doxycyclin is a broad-spectrum antibiotic extensively used for lyme disease, brucellosis, and several rickettsial infections. It is the drug of choice in the treatment of periodontitis which is one of the main causes of tooth loss. The formation of periodontal pockets between the teeth and gums promotes the growth of aggressive forms of bacteria ${ }^{7}$. However, the periodontal pockets are easily accessible from the oral cavity and so provide a convenient site for local treatment by drug delivery systems. In an earlier study we have shown the controlled release of doxycycline by BCP ceramics ${ }^{8}$ which is a two phase system. The present study is focused on the preparation of single phase CDHA microspheres; its characterization and doxycycline release profiles for the treatment of periodontal diseases.

\section{Materials and Methods}

High purity calcium hydroxide $\left[\mathrm{Ca}(\mathrm{OH})_{2}\right]$ and diammonium hydrogen orthophosphate (DAP, $(\mathrm{NH} 4)_{2} \mathrm{HPO}_{4}$ ) were used for the preparation of CDHA powder (Sigma Aldrich Chemicals, USA). Pharmaceutical grade low viscosity paraffin oil, gelatin (bovine source, SD fine chemicals, India), and the antibacterial drug doxycyclin hyclate (Periostat, Ranbaxy Pharmaceuticals, India) were procured locally. The CDHA powder was synthesized by the microwave method. Weighed amounts of the starting granules were dissolved in water and the DAP solution was added to the calcium hydroxide solution under stirring conditions. The solution was then exposed to microwave irradiation in a microwave oven (BPL, India). The product was then filtered and dried in an oven at $100^{\circ} \mathrm{C}$. Three different $\mathrm{CDHA}$ systems were prepared by varying the $\mathrm{Ca} / \mathrm{P}$ molar ratio to $1.56,1.61,1.64$ and the samples have been coded as CD156, CD161, and CD164 respectively.

An appropriate aqueous solution of gelatin content was prepared at $30^{\circ} \mathrm{C}$. Fine $\mathrm{CD} 161$ powder were added to the above solution and the gelatin slurry with CDHA powder was dispersed in light paraffin oil in an analytical flask by stirring with a glass paddle stirrer. The rpm of the stirrer and the time of stirring were optimized to form microspheres. The precipitated microspheres were washed in acetone followed by ethanol and dried in air. The gelatin bound beads were heated for an hour at $550^{\circ} \mathrm{C}$ to burn off the gelatin and the microspheres were then thoroughly washed in distilled water to remove any unburned gelatin and dried in an oven. The microspheres formed with $4 \%(4 \mathrm{~g}$ in $100 \mathrm{ml})$, $6 \%(6 \mathrm{~g}$ in $100 \mathrm{ml})$, and $8 \%(8 \mathrm{~g}$ in $100 \mathrm{ml})$ gelatin have been coded as 4CD161MS, 6CD161MS, and $8 C D 161 M S$ respectively in the text. The same process was repeated for other CDHA powder and the microspheres were coded accordingly as shown above.

The synthesized CDHA powder and microsphere samples were characterized by $\mathrm{X}$-ray powder diffraction (XRD) method (Shimadzu XDD1 X-Ray diffractometer, reflection mode, Japan) using $\mathrm{CuK} \propto$ radiation. The morphology of the $\mathrm{CDHA}$ granules and microspheres were observed under a scanning electron microscopy (JOEL JSM 5410 \& JSM 5300, Japan). A few milligram of dried CDHA sample was deposited on a black adhesive tape, vacuum coated with gold film for 15 minutes and analyzed directly. The surface area of the microspheres was determined by the triple-point BET method (Sorptomatic 1990, USA) with nitrogen as the adsorbate gas and helium as an inert non-adsorbable carrier.

TheeffectofpHontheencapsulation ofdoxycyclin into CDHA microsphere was initially carried out in phosphate buffer saline (PBS) at room temperature to determine the suitable $\mathrm{pH}$ for loading of the maximum amount of the drug. Buffers of varying $\mathrm{pH}$ were prepared and $10 \mathrm{mg}$ of the drug was dissolved in the $\mathrm{pH}$ solutions. The CDHA microspheres were suspended in the above phosphate buffers for three hours. The microspheres were then separated and the concentration of the doxycyclin was measured using UV-Visible spectrophotometry (Varian Cary 5E UV-VIS-NIR Spectrophotometer, USA) with a 1-cm path length cuvette. Subsequently the loading was carried out at the optimal $\mathrm{pH}$ of the buffer. A typical UV visible spectrum of the doxycyclin hyclate has four characteristic peaks in the UV region as shown in Figure 1 , and of the 4 peaks the very prominent peaks at $270 \mathrm{~nm}$ and $350 \mathrm{~nm}$ were monitored for the drug loading profile. 


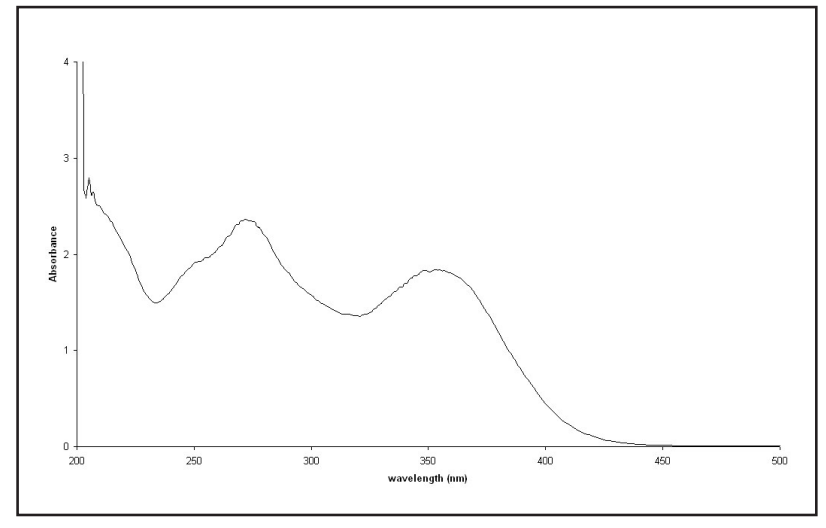

Figure 1. Typical UV-visible spectrum of doxycycline.

To calculate the amount of drug loading, initially $10 \mathrm{mg}$ of CDHA microspheres were immersed in $10 \mathrm{ml}$ of PBS containing $10 \mathrm{mg}$ of doxycyclin for 24 hours. The microspheres were separated by centrifugation and dried at room temperature for 48 hours. The amount of drug absorbed was calculated spectrometrically by finding the difference in doxycyclin concentration in the loading buffer, before, and after loading by using the equation:

$$
\text { Percentage drug loading }=((A-B) / A) * 100
$$

where $A$ and $B$ represent the initial and final drug concentrations of the buffer solution respectively and by varying the concentration of drug and the CDHA microspheres the maximum amount of loading of the drug was obtained.

The in vitro studies were carried out by soaking the drug loaded microspheres in sodium phosphate buffer solution as the medium for drug release at $\mathrm{pH}$ 7.4 and $37^{\circ} \mathrm{C}$. Triplicate samples of $10 \mathrm{mg}$ of granules were suspended in $20 \mathrm{ml}$ phosphate buffer in glass tubes. The tubes were placed in a bench top constant temperature water bath. At regular intervals of time, solutions were withdrawn and drug release profiles were obtained by the UV-visible spectrophotometry as mentioned above. The doxycyclin release from the specimen to the buffer was determined by measuring the absorbance values at the maximum observed at $\lambda=270 \mathrm{~nm}$. The spectra were recordedfor every 30 minutes until there was no change in the subsequent values.
In vitro solubility studies of CDHA microspheres were carried out in phosphate buffer of $\mathrm{pH} 7.4$ at $37^{\circ} \mathrm{C}$. Dissolution experiments were performed by using $0.1 \mathrm{~g}$ of the pure microspheres without drug loading in $50 \mathrm{ml}$ of buffer of $\mathrm{pH}$ 7.4. Each tube containing buffer with the microspheres was maintained in a constant temperature water bath. The change in the $\mathrm{pH}$ of the buffer solution containing the microspheres was recorded at regular intervals of time during the dissolution experiments. All the experiments were performed in triplicate, by running three parallel independent measurements simultaneously. The weight loss was calculated by recording the initial and final weights of the microspheres. This weight loss indicates the percentage dissolution of the microspheres of various compositions.

\section{Results and Discussion}

The XRD pattern of the finally powdered oven dried CDHA samples are shown in Figure 2. The patterns essentially look similar to each other with major characteristic diffraction peaks corresponding to CDHA phase ${ }^{6}$. Although they have similar XRD pattern, the sample crystallinity as shown by the sharpness of the peak varies with the $\mathrm{Ca} / \mathrm{P}$ ratio. The sample CD156 has the lowest crystallinity among the others.

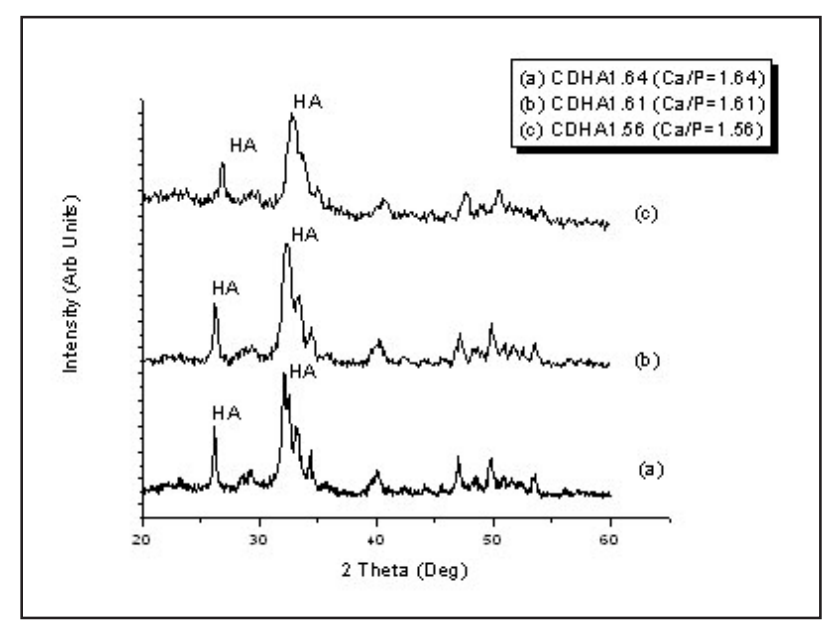

Figure 2. XRD of CD164 (a), CD161 (b), and CD156 (c) powder. 
The cell parameters of CDHA (Table 1) were calculated by least squares fit method and is found to decrease with increasing $\mathrm{Ca} / \mathrm{P}$ ratio as expected for CDHAs.

Table 1. Unit cell parameters and surface area of CDHA samples.

\begin{tabular}{cccc}
\hline \multirow{2}{*}{$\begin{array}{c}\text { CDHA } \\
\text { sample(Ca/P) }\end{array}$} & \multicolumn{2}{c}{ Cell parameters $(\mathrm{nm})$} & \\
\cline { 2 - 3 } & $\mathbf{a}=\mathbf{b}$ & $\mathbf{c}$ & $\begin{array}{c}\text { BET Area } \\
\left(\mathbf{m}^{2} / \mathbf{g m}\right)\end{array}$ \\
\hline 1.56 & 0.943 & 0.688 & 38 \\
1.61 & 0.943 & 0.686 & 72 \\
1.64 & 0.941 & 0.686 & 54 \\
\hline
\end{tabular}

Table 2. Surface area and drug release percentages of CDHA microspheres.

\begin{tabular}{ccccccc}
\hline $\begin{array}{c}\text { Gelatin } \\
\text { concen- } \\
\text { tration }\end{array}$ & \multicolumn{2}{c}{ CDHA1.56MS } & \multicolumn{2}{c}{ CDHA1.61MS } & \multicolumn{2}{c}{ CDHA1.64MS } \\
\cline { 2 - 7 } & $\begin{array}{c}\text { BET } \\
\text { Area } \\
\left(\mathrm{m}^{2} / \mathrm{g}\right)\end{array}$ & $\begin{array}{c}\text { \% Drug } \\
\text { release }\end{array}$ & $\begin{array}{c}\text { BET Area } \\
\left(\mathrm{m}^{2} / \mathrm{g}\right)\end{array}$ & $\begin{array}{c}\text { \% Drug } \\
\text { release }\end{array}$ & $\begin{array}{c}\text { BET } \\
\text { Area } \\
\left(\mathrm{m}^{2} / \mathrm{g}\right)\end{array}$ & $\%$ Drug release \\
\hline 4 & 28 & 39 & 40 & 53 & 19 & 24 \\
6 & 35 & 48 & 75 & 80 & 25 & 29 \\
8 & 31 & 46 & 53 & 65 & 22 & 26 \\
\hline
\end{tabular}

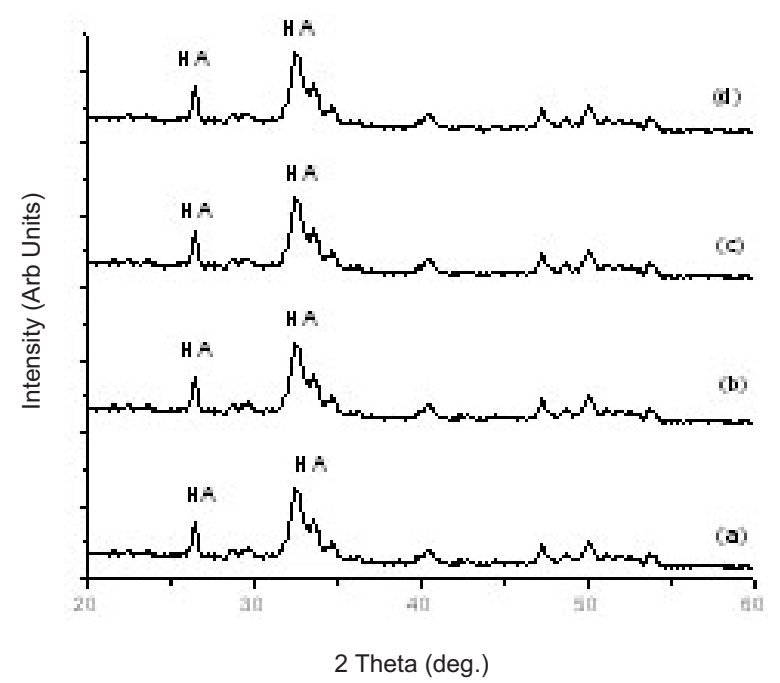

Figure 3. XRD of CD161 powder (a) 4CD161, (b) 6CD161 (c) and 8CD161 (d) microspheres.

The specific surface area of the microspheres determined using BET isotherm is listed in Table 2.
The surface area has been found to vary between 19 to $75 \mathrm{~m}^{2} / \mathrm{g}$ depending on the $\mathrm{Ca} / \mathrm{P}$ ratio and gelatin concentration as similar to BCP microspheres ${ }^{8}$. The microspheres formed using $6 \%$ gelatin show larger surface area compared to other CDHA microspheres formed with different gelatin content. Similarly, the $\mathrm{CDHA}$ microspheres corresponding to $\mathrm{Ca} / \mathrm{P}$ ratio of 1.61 have larger surface area than the CDHA microspheres with different $\mathrm{Ca} / \mathrm{P}$ ratios. Hence, further characterization of CD161 microspheres has been carried out.

The typical XRD pattern of the CD161 microspheres formed with various amounts of gelatin is shown in Figure 3. The patterns look similar to that of the starting CDHA powder as also shown in the same figure for comparison. This indicates the stability of the CDHA microspheres formed through heating at $650^{\circ} \mathrm{C}$ to remove the gelatin. The broad characteristic XRD peaks indicate the microcrystalline nature of the samples.

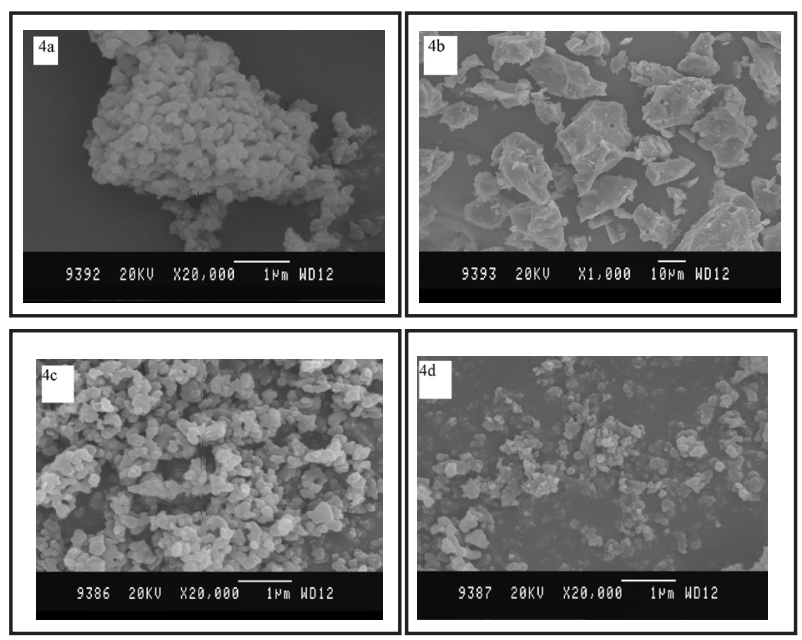

Figure 4.SEM micrographs of CD161 powder (a), 4CD161MS (b), 6CD161MS (c) and 8CD161MS (d) microspheres.

The SEM micrograph of the powder and microspheres of CD161 is shown in Figure 4a-d. The morphology of the CD161 powder is spherulite and mostly agglomerated. There is a distinct difference in the morphology of the microparticles corresponding to a transition from irregular shape to spherical shape with smooth surfaces as a function of gelatin content. Although, the 6CD161MS sample 
indicates considerable agglomeration (Figure 4(b)), the microspheres are uniform and more spherical in contrast to other samples.

The dissolution studies of the CDHA microspheres for all the $\mathrm{Ca} / \mathrm{P}$ ratios prepared were carried out to determine the solubility of the resorbable bioceramics used. Both the $\mathrm{pH}$ variation of the buffer solution containing the microspheres and the weight of the microspheres were determined as a function of immersion time.
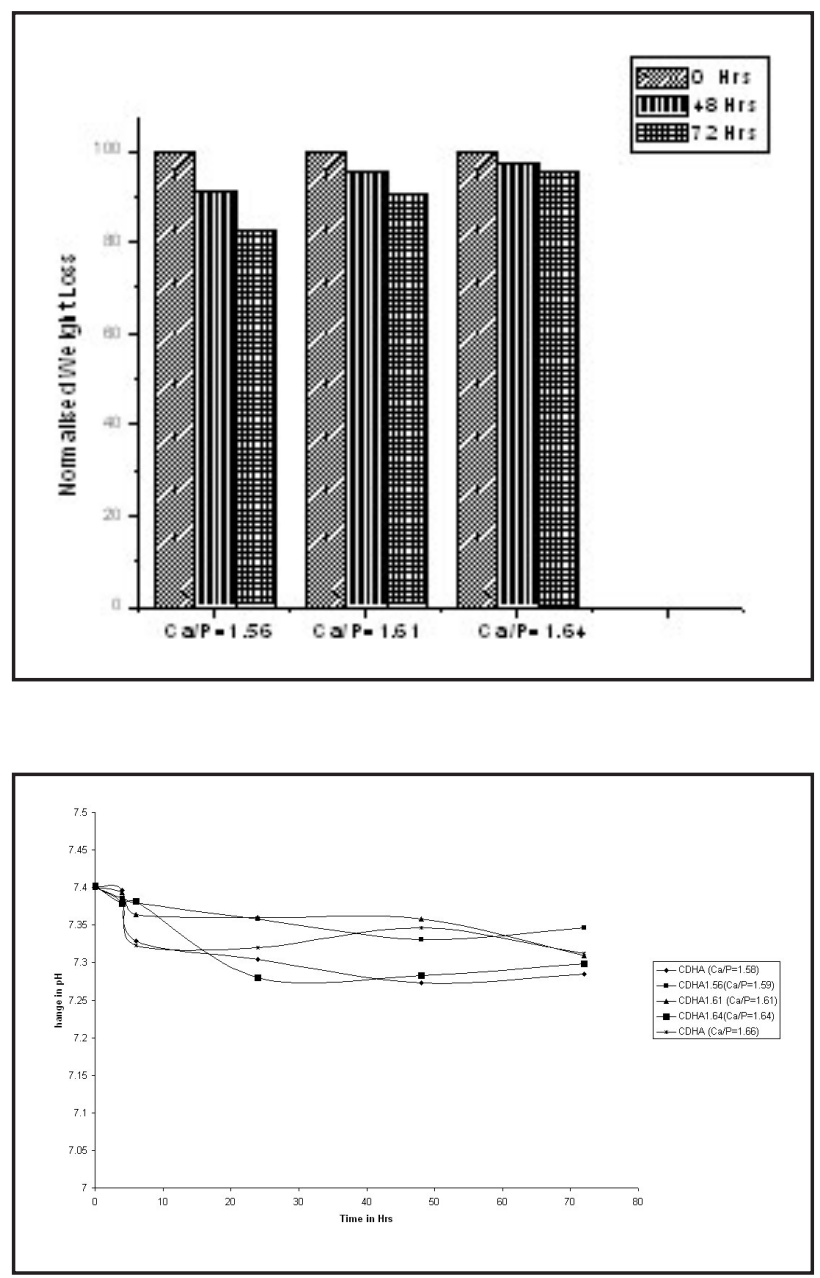

Figure5. pH variation of CDHA microspheres containing buffer as a function of time (a) and weight loss of the CD156, CD161 and CD164 microspheres (b).

The $\mathrm{pH}$ variation of the buffer solution containing the CDHA samples is shown in Figure 5a. All the samples show drastic decrease of $\mathrm{pH}$ values initially followed by nearly a constant value depending on the $\mathrm{Ca} / \mathrm{P}$ ratios. However the CD161 and CD166 microspheres show an increase in $\mathrm{pH}$ after 50 hours. The overall trend in dissolution has been correlated with the nonstoichiometric behavior of CDHA ceramics based on a chemical model ${ }^{9}$. In this model, the initial stage of apatite dissolution consists of calcium detachment from the surface and subsequent detachment of phosphates from the surface ${ }^{10}$. Weight loss of the microspheres for all the three types of 6CDMS samples were observed during the dissolution studies (Figure 5b). The dissolution increases with a decrease in the $\mathrm{Ca} / \mathrm{P}$ ratio as expected since more stable $\mathrm{HA}$ has a $\mathrm{Ca} / \mathrm{P}$ ratio of 1.66 while that of resorbable TCP is 1.5. Dissolution efficiency depends on the available surface area and the specific surface area of the 6CDMS microspheres varies from 25 to $75 \mathrm{~m}^{2} / \mathrm{g}$. However, this parameter was found to be not related to $\mathrm{Ca} / \mathrm{P}$ molar ratio and so the dissolution behavior.

The drug intake by the microspheres depends on the $\mathrm{pH}$ conditions of the buffer solution. The $\mathrm{pH}$ of 7 was found to have maximum loading of the drug and hence, selected for release study. The doxycycline seems to be stable at the various pHs studied. Higher amount of loading was shown by the CD161 microspheres.

The doxycycline release profiles of CD161 microspheres are shown in Figure 6a. All the microspheres exhibits similar release profiles with a gradual increase initially reaching a maximum value and then nearly a constant release profile. A maximum amount of about $80 \%$ drug release was observed for the 6CD161MS while 8CD161MS showed about $60 \%$ drug release. Both the 6 CD161MS and $8 \mathrm{CD} 161 \mathrm{MS}$ microspheres showed the maximum release at the ninth hour. As the 6CD161MS were relatively found to be uniformly spherical with a smoother surface, the observed release profile has been attributed to the surface bound drug $^{8}$. It also has more surface area as indicated in Table 2 . Hence, more amount of drug could have been absorbed on the 6CD161MS, which results in the maximum amount of drug release. The 4CD161MS were found to be larger which results in lesser amount of drug absorption and release at a shorter time interval. The results thus suggest optimum morphology, size, 
and presence of micropores in 6CD161MS leading to the observed release profile.
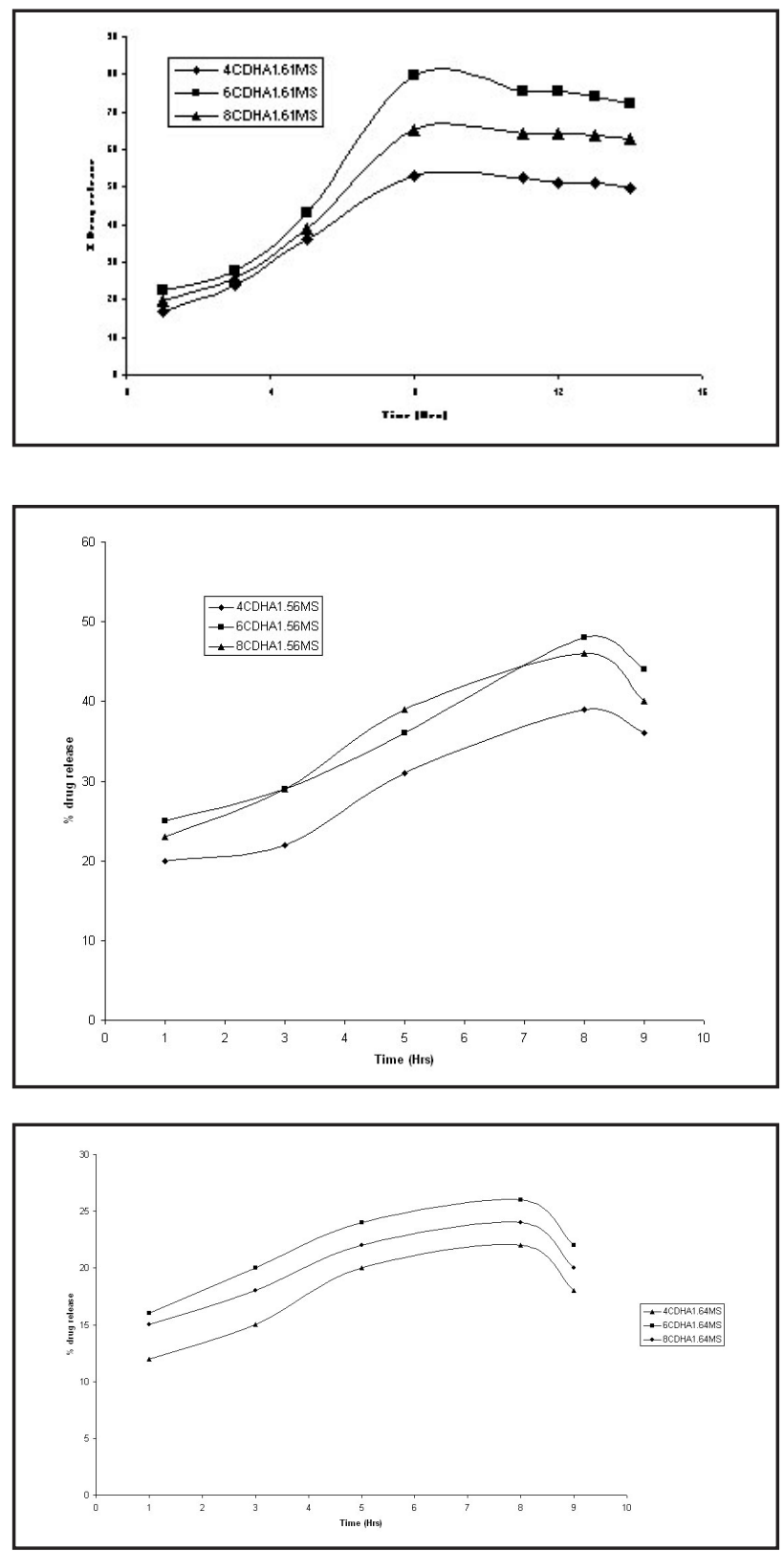

Figure 6. In vitro drug release profile of CD161 (a), CD156 (b), and CD164 (c) microspheres.

Figures $6 \mathrm{~b}$ and $6 \mathrm{cshow}$ the in vitro release profiles of CD156 and CD164 microspheres respectively. The profiles are similar to those obtained for the CD161 microspheres. However, the maximum drug release is much lower (50\% in CD156MS and 23\% in CD164MS respectively) than that obtained for the CD161MS (80\% in CD161MS). The above results correlated with the surface area measurements as indicated in Table 2. The $6 C D 161 \mathrm{MS}$ has the highest surface area, which leads to more loading and ultimately better release characteristics. Compared to the other samples, the maximum amount of release ( $80 \%$ of drug) similar to a burst release of this sample may be due to the liberation of surface bound drug as indicated earlier.

The open pore content in the microsphere is dependent on the gelatin concentration in starting $\mathrm{CDHA} /$ gelatin mixture. Although the $8 \mathrm{CD} 161 \mathrm{MS}$ microspheres were found to be porous, only $60 \%$ of the drug was released. This indicated that the size of the pores might be larger so as to not retain the doxycyclin drug. The 4CD161MS microspheres were found to be of larger size and without much pores and so these features result in lesser amount of drug absorption and release compared to other microspheres. The results thus suggest optimum morphology, size and presence of micropores in $6 C D 161 \mathrm{MS}$ leading to the observed release profile. As the behavior of a particulate in the body depends on its morphology and porosity, the constant release of the drug thus can be controlled by appropriate selection of the microspheres by optimizing the $\mathrm{Ca} / \mathrm{P}$ ratios to have the required features.

\section{Conclusion}

CDHA microspheres with optimum pore content and morphology were prepared. Doxycycline loading around physiological conditions leads to higher amount of loading. Maximum drug release was observed for the microspheres formed using $6 \%$ gelatin. Release pattern strongly depends on the morphology of CDHA microspheres and an optimum release up to $80 \%$ has been recorded. The morphological features of the microsphere were found to correlate well with the in vitro doxycycline release and the surface area measurements (maximum in the case of $6 \%$ gelatin). 


\section{References}

1. Di Silvio L, Bonfield W. 1999. Biodegradable drug delivery system for the treatment of bone infection and repair. J Mater Sci: Mater Med 10:653-8.

2. Mansho I, Wenyi Y, Takaaki A, Akira O, Naoki K. 1998. Synthesis of antibioticloaded interporous hydroxyapatite blocks by vacuum method and invitro release testing. Biomaterial 19:817-9.

3. Alkhraisat $\mathrm{MH}$, Rueda C, Cabrejos-Azama J, Lucas-Aparicio J, Mariño FT,García-Denche JT, Jerez LB, Gbureck U, Cabarcos EL. 2010. Loading and release of doxycycline hyclate from strontium-substituted calciumphosphate cement. Acta Biomateralia 6:1522-8.

4. Thomazeau H, Langlais F. 1997. Antibiotic release by tricalcium phosphate bone implantation, in vitro and in vivo pharmacokinetics of different galenic forms. Chirurgie 121:663-6.

5. Yamamura K, Iwata H, Yotsuyanagi T. 1992. Synthesis of antibiotic-loaded hydroxyapatite beads and in vitro drug release testing. J Biomed Mater Res 26:1053-64.
6. Siddharthan A, Seshadri SK, SampathKumar TS. 2004. Microwave accelerated synthesis of nanosized calcium deficient hydroxyapatite. J Mater Sci: Mater Med 15: 1279-84.

7. Bromberg LE, Debra KB, Phillip MF. 2001. Novel periodontal drug delivery system fortreatment of periodontitis. Journal of Controlled Release 71: 251-9.

8. Sunita PV, Sampath Kumar TS. 2008. BCP ceramic microspheres as drug delivery carriers -synthesis, characterization and doxycycline release. J Mater Sci: Mater Med 19: 283-90.

9. Elena $M$, Alexandre MR, Nilce CCR, Gloria AS, Josin CM, Gustavo TM. 2003. Dissolution of calcium-deficient hydroxyapatite synthesized at different conditions. Materials Characterization 50:203-7.

10. Monteiro MM, Rocha NCC, Rossi AM, Soares GA. 2003. Dissolution properties of calcium phosphate granules with different compositions in simulated bodyfluid. J Biomed Mater Res A 65:299-305. 